\title{
Design and Optimization of EPON Link
}

\author{
Priyanka Sehgal \\ M. Tech Research scholar \\ (ECE) \\ Amritsar College of \\ Engineering \& Technology \\ Punjab, India
}

\author{
Gaurav Soni \\ Associate Professor \\ Department of Electronics \& \\ Communication Engineering \\ Amritsar College of \\ engineering \& Technology \\ Punjab, India
}

\author{
Narinder Sharma \\ Associate Professor(HOD) \\ Department of Electronics \& \\ Communication Engineering \\ Amritsar College of \\ engineering \& Technology \\ Punjab, India
}

\begin{abstract}
EPON (Ethernet passive optical networks) is a new technology that is inexpensive, simple and capable of delivering bundled voice, data and video services to an end user subscriber over a single network. The Proposed EPON link is designed using the Optisystem software by taking transmitter and receiver and optical fiber to send and receive bundled data from each other. The link is further improved by selecting wavelength, transmission distance, transmission capacity and selecting different modulation schemes to make the EPON link more efficient.
\end{abstract}

\section{Keywords}

EPON (Ethernet passive optical networks), OLT (optical line terminal), ONU (optical network unit), ODN (optical distribution network)

\section{INTRODUCTION}

In recent decades, the telecommunication backbone system has been replaced from time to time while more fibers are set and products of larger sizes joined in to utilize. But have you been aware that entry system, the copper line remains the primary choice. The remarkable upsurge in Net companies has discovered the shortage of the entry network's capacity. The entry system that is named "last mile" still remains the bottleneck between high-speed LANs and the high-capacity backbone network[1-4].The absolute most widely deployed "broadband" solutions today are DSL and CM networks. Though they are changes when compared with $56 \mathrm{kbps}$ dialup lines, they are however struggling to supply enough bandwidth for emerging services such as like video-ondemand (VOD), interactive gambling, or two-way video conferencing. A whole new type of access technology is required for enough time with following functions: cheap, easy, upgradeable, and to handle to supply provided style, information and video services. EPON, which merges the low-cost Ethernet technology and low-cost optic network structure, is the higher representative of the future-oriented next technology access network technologies.As early as in November 2000, about 200 professionals from 80 businesses shaped a examine group of IEEE and this party was chartered with increasing present Ethernet technology into client access area. Then in September 2001, this examine party is officially named as Ethernet in the First mile (EFM) examine party and is dedicated to instituting EPON requirements within the structure of the IEEE 802.3 protocol. In August 2004, the IEEE 802.3ah typical is accepted by IEEE Standard Panel and then officially released. EPON is on the foundation of the Ethernet standard, unlike different

PON technologies, which are on the foundation of the ATM standard. Letting you utilize economies-of-scale of Ethernet, and provides simple, easy-to-manage connectivity to Ethernet-based, IP equipment, both at the customer premises and at the central office. Similar to different Gigabit Ethernet press, it's well-suited to move packetized traffic, which is dominant at the accessibility layer, in addition to timesensitive style and movie traffic and therefore has $2 \mathrm{~N}$ optical transceivers. Curb-switched Ethernet uses one trunk fiber and therefore saves fiber and room in the Central office (CO). Nevertheless it uses $2 \mathrm{~N}+2$ optical transceivers and wants electric power in .EPON also uses just one single trunk fiber and therefore reduces fibers and room in the $\mathrm{CO}$, and also only uses N+1optical transceivers. It will take number electric power in the field. The drop throughput may be across the point rate on the trunk link. EPON may help downstream transmitted such as like video. The IEEE 802.3ah EPON specification identifies Multi-Point Get a handle on Project (MPCP), Point-to-Point Emulation (P2PE), and two 1490/1310 nm PMDs for 10and $20 \mathrm{~km}$, required to construct an EPON system. Normal EPON[7]-based programs may possibly include added characteristics above the IEEE 802.3ah standard, including safety, verification and dynamic bandwidth allocation.

\section{ARCHITECTURE OF ETHERNET PASSIVE OPTICAL NETWORKS}

A typical EPON system is composed of OLT, ONU, and ODN

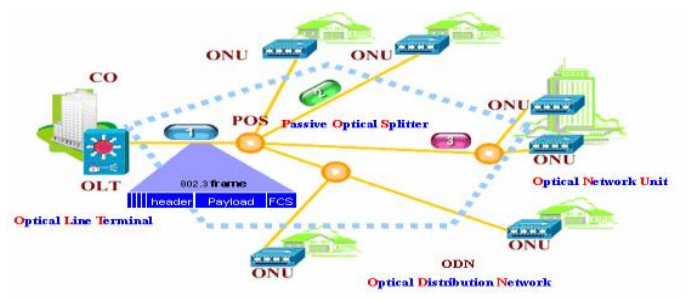

Figure 1 EPON Network Architecture

OLT (Optical line Terminal) resides in the Key Company (CO) and links the optical network to the metropolitan-area network or wide-area network, also referred to as the backbone or long-haul network. OLT is equally a transition or router and a multi-service program which materials EPONoriented visual interfaces. Sort network assembling and accessibility functions, OLT may also accomplish bandwidth assignments, network security and administration configurations on the basis of the customers' different QoS / SLA requirements.The ONU (Optical network Unit) is located possibly at the end- OLT (Optical line Terminal) resides in the Key Company (CO) and links the optical network to the metropolitan-area network or wide-area network, also referred to as user area or at the suppress and offers visual interfaces which are attached with the OLT and support interfaces at 
people's part such as for instance like voice, data and video.The ODN (optical distribution network) is an aesthetic circulation network and is principally contains more than one inactive visual splitters which join the OLT and ONU. Their purpose is always to separate downstream indicate in one fiber into a few materials and mix visual upstream. Visual splitter is just a easy system which needs no power and could work in a all-weather environment. The conventional splitters have a breaking ratio of $2,4,8,16$ or 32and connect to each other.

\section{LINK DESIGN}

The following hyperlinks were created in opti system 13. All of the variables for the planning are taken based on IEEE 802.3 ah standards. We've connected five routes with $1 \mathrm{~nm}$ spacing. Here nine ONUs are associated with a central company (OLT) via an optical fiber accessibility system using WDM technology. In downstream path five channels $1550 \mathrm{~nm}$, $1551 \mathrm{~nm}, 1552 \mathrm{~nm}, 1553 \mathrm{~nm}$ and $1554 \mathrm{~nm}$ wavelengths with 1 $\mathrm{nm}$ spacing is mixed through WDM MUX and then transmitted via bidirectional visual fiber. WDM MUX is introduced to mix these carriers for the more transmission. The productivity of WDM MUX is transmitted through bidirectional optical fiber and then productivity is separated by using 1:8 splitters to eight ONUs. The received indicate after WDM DEMUX is fed in to a PIN photo detector. Different analyzers equally at transmitter and recipient side are attached for the analysis. After ONU1, we have attached five $3 R$ regenerators and $B E R$ analyzers for result examination. The fig 2 represents the link diagram of EPON following RZ modulation scheme.

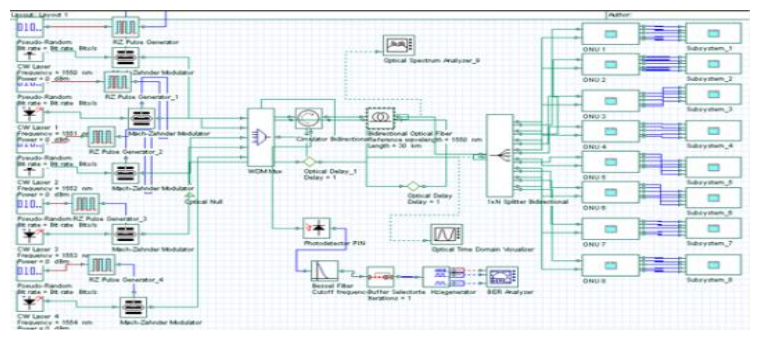

Fig 2 Proposed EPON link

\section{RESULTS}

The following results are optimized from the designed link having different number of users at the receiver end.

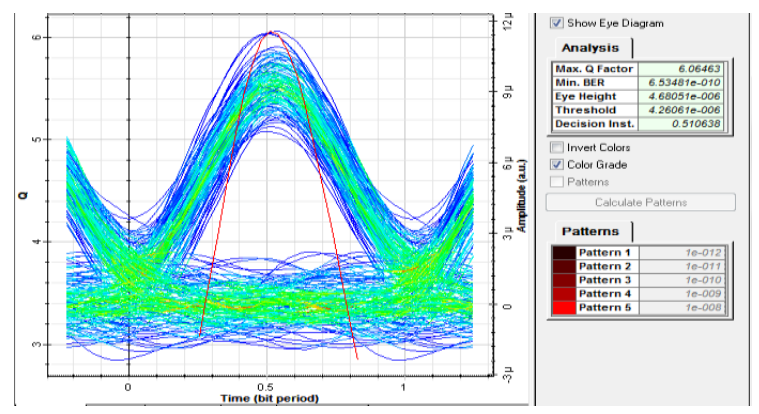

Fig 3. Eye diagram of downstream at Rx channel 2

In the above Fig 3, it represents the RZ eye diagram in downstream at receiver channel 2 . In this figure $\mathrm{x}$-axis represents the time (bit period) and y-axis represents the Qfactor. The maximum Q-factor obtained at the receiver channel 2 is 6.06463 and minimum BER obtained is $6.53481 \mathrm{e}-010$.

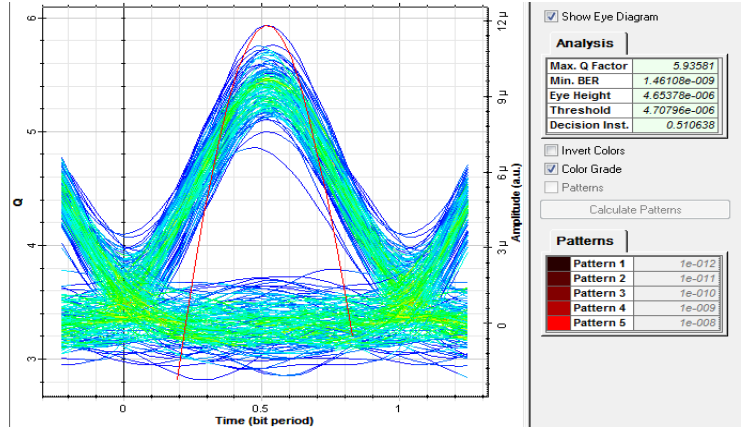

Fig 4 Eye diagram of downstream at Rx channel 40

In the above Fig 4, it represents the RZ eye diagram in downstream at receiver channel 40 . In this figure $\mathrm{x}$-axis represents the time (bit period) and y-axis represents the Qfactor. The maximum Q-factor obtained at the receiver channel 40 is 5.93581 and minimum BER obtained is $1.46108 \mathrm{e}-009$.

\section{CONCLUSION}

EPON (Ethernet passive optical networks) is a new technology which is required to meet the requirements of high data-rate; video-on-demand and other services like two way conferencing. It accessed by using Optical line terminal (OLT) which is located at the premises of the central office and Optical network units (ONUs) which are located at various user ends. OLT access the various ONUs together by using the point to multi-point protocol. EPONS follows full duplex mode and follows point to multi-point topology. The results are optimized at each user end using BER analyzers. The modulation scheme of RZ is used in the designed links and the optimized results show that the EPON link with RZ modulation scheme performs satisfactorily.

\section{REFERENCES}

[1] Baljeet Kaur, Ajay K. Sharma, Vinod Kapoor; "On WDM RoF-EPON link using OSSB transmission with and without square root module"; Optik 124 (2013) $1334-1337$

[2] Baljeet Kaur, Ajay K. Sharma, Vinod Kapoor; "Performance enhancement with square root module for WDM RoF-EPON link"; Optik 124 (2013) 967-971

[3] Baljeet Kaur, Ajay K. Sharma, Vinod Kapoor; "Performance improvement on OVSB based WDM RoFEPON link using SOA with DCF and FBG"; Optik 125 (2014).

[4] Baljeet Kaur, Ajay K. Sharma, Vinod Kapoor; "On WDM RoF-EPON link using OSSB transmission with and without DCF + FBG”; Optik 125 (2014).

[5] Vishal Sharma, Shivani Sharma; "Hybrid multiplexed OFDM-DWDM-EPON access network"; Optik 125 (2014).

[6] Access control for Ethernet passive optical networks: an overview." Communications Magazine, IEEE 43, no. 2 (2005): 145-150.

[7] C.-J. Chae et al., "Optical CSMA/CD Media Access Scheme for Ethernet over Passive Optical Network," IEEE Phot. Tech. Lett., vol. 14, no. 5, May 2002, pp. 711-13. 